\title{
Uma Abordagem Computacional do Contorno* de Grafos
}

\author{
$\underline{\text { Alonso L. S. de Oliveira }}^{\dagger} \quad$ Danilo Artigas \\ Instituto de Ciência e Tecnologia, \\ Universidade Federal Fluminense, \\ 28895-532, Rio das Ostras, RJ \\ E-mail: alonsoleonardo@id.uff.br, daniloartigas@puro.uff.br
}

\section{$\underline{\text { RESUMO }}$}

Neste trabalho consideramos um problema relacionado a convexidade geodésica em grafos. O conceito de convexidade em estruturas discretas foi estendido a partir do conceito para matemática contínua.

Os grafos adotados são finitos, simples e conexos. Seja $G$ um grafo, denotamos seu conjunto de vértices por $V(G)$ e o conjunto de arestas por $E(G)$, onde $n$ é o número de vértices de $G$ e $m$ é o número de arestas de $G$. O intervalo fechado $I[S]$ de um conjunto $S \subseteq V(G)$ é o conjunto de todos os vértices que se encontram em algum caminho mínimo entre pares de vértices de $S$, incluindo os vértices em $S$. Um conjunto $S \subseteq V(G)$ é geodésico se $I[S]=V(G)$. A distância $d(v, w)$ entre dois vértices $v, w \in V(G)$ é o número de arestas no caminho mínimo entre $v$ e $w$. A excentricidade ecc $(v)$ de um vértice $v$ é o máximo de $d(v, w)$ para todo vértice $w \in V(G)$. O diâmetro $\operatorname{diam}(G)$ de $G$ é máximo ecc $(v)$ para todo vértice $v \in V(G)$. O contorno $C t(G)$ de $G$ é o conjunto dos vértices cuja excentricidade é maior ou igual que a de seus vizinhos.

Neste trabalho consideramos o problema de determinar se o conjunto de contorno de um grafo é geodésico. Este problema foi definido em [3], onde os autores apresentaram o grafo $G_{1}$ da Figura 1, observe que $C t\left(G_{1}\right)=\{a, b, c\}$ e $I\left[C t\left(G_{1}\right)\right]=V(G) \backslash\{d\}$, logo $C t\left(G_{1}\right)$ não é um conjunto geodésico. Além disso, provaram que o contorno dos grafos pertencentes a classe distância hereditária é geodésico. Em [2], os autores apresentaram o grafo $G_{4}$ da Figura 1, que é obtido estendendo-se o grafo $G_{1}$, e analogamente a $G_{1}$ temos que $C t\left(G_{4}\right)=\{a, b, c\}$ e $I\left[C t\left(G_{4}\right)\right]=V(G) \backslash\{d\}$. Ainda mais, $G_{4}$ é um grafo de permutação. Por fim, provaram que para todo grafo cordal o contorno é geodésico e apresentaram um esquema de classes de grafos classificadas em 3 tipos: classes onde todo grafo possui o contorno geodésico; classes onde nem todo grafo possui o contorno geodésico; e classes em aberto, onde a resposta não era conhecida. Em [1], todos as classes mencionadas como em aberto em [2] receberam respostas definitivas e, além disso, os autores apresentaram um novo grafo cujo contorno não é geodésico.

Os 3 artigos mencionados no parágrafo anterior apresentaram, em 8 anos, apenas 3 grafos diferentes tais que o contorno não é geodésico. A dificuldade de obtenção desses exemplos se deve, primeiramente, porque tais grafos não são comuns, mas principalmente pelo grande trabalho necessário para calcular manualmente as excentricidades dos vértices de $G$, determinar $C t(G)$ e verificar se $C t(G)$ é geodésico. Esta dificuldade torna a abordagem computacional promissora para análise do problema, e este é o foco deste trabalho.

Dado um grafo $G$, o algoritmo implementado para verificar se $C t(G)$ é geodésico consiste em: calcular a excentricidade de cada vértice de $G$, aplicando $n$ vezes o algoritmo de busca em largura; determinar para cada vértice $v \in V(G)$ se $v \in C t(G)$, comparando a excentricidade de $v$ com a de seus vizinhos; e testar se $I[C t(G)]=V(G)$, verificando para cada vértice $v \in V(G)$ se existe $x, y \in C t(G)$ tal que $v$ se encontra em um caminho mínimo entre $x$ e $y$. O algoritmo descrito tem complexidade

\footnotetext{
${ }^{*}$ Parcialmente financiado por Proppi/PDI/UFF

†bolsista de Iniciação Científica PIBIC/UFF
} 
$O\left(n^{3}\right)$, nossa ideia central é analisar conjuntos de grafos, com alguma propriedade específica, e verificar se neste conjunto existem grafos cujo contorno não é geodésico. Por exemplo, verificar se existe um grafo $C t(G)$ de 10 vértices tal que $I[C t(G)] \neq V(G)$. No entanto, o número total de grafos não isomorfos de 10 vértices é muito grande, portanto acrescentamos alguns testes ao algoritmo para melhorar seu desempenho. Alguns desses testes foram extraídos de [1], onde os autores provaram que o contorno de um grafo $G$ qualquer é geodésico se $\operatorname{diam}(G) \leq 4$ ou $\operatorname{diam}(G) \leq 7$ para um grafo $G$ bipartido. Além disso, desenvolvemos o resultado a seguir.

Teorema 1. Seja $G$ um grafo e $S \subseteq V(G)$ um conjunto tal que $C t(G) \subseteq S$ e $|S| \geq V-3$, então $S$ é geodésico.

Como consequência do Teorema 1, se $|C t(G)| \geq V-3$, então $C t(G)$ é geodésico. Adicionando este teste e aqueles mencionados no parágrafo anterior obtemos o algoritmo que utilizamos para a obtenção dos resultados computacionais deste trabalho. Inicialmente, consideramos grafos com um número limitado de vértices e, testando todos os casos, descobrimos um limite inferior para o número de vértices de grafos cujo contorno não é geodésico.

Teorema 2. Se $n \leq 9$, então $C t(G)$ é geodésico.

Também verificamos quantos grafos de 10 vértices não possuem o contorno geodésico. E identificamos quais são estes grafos.

Teorema 3. Existem 4 grafos com 10 vértices cujo contorno não é geodésico. Estes grafos são os da Figura 1.
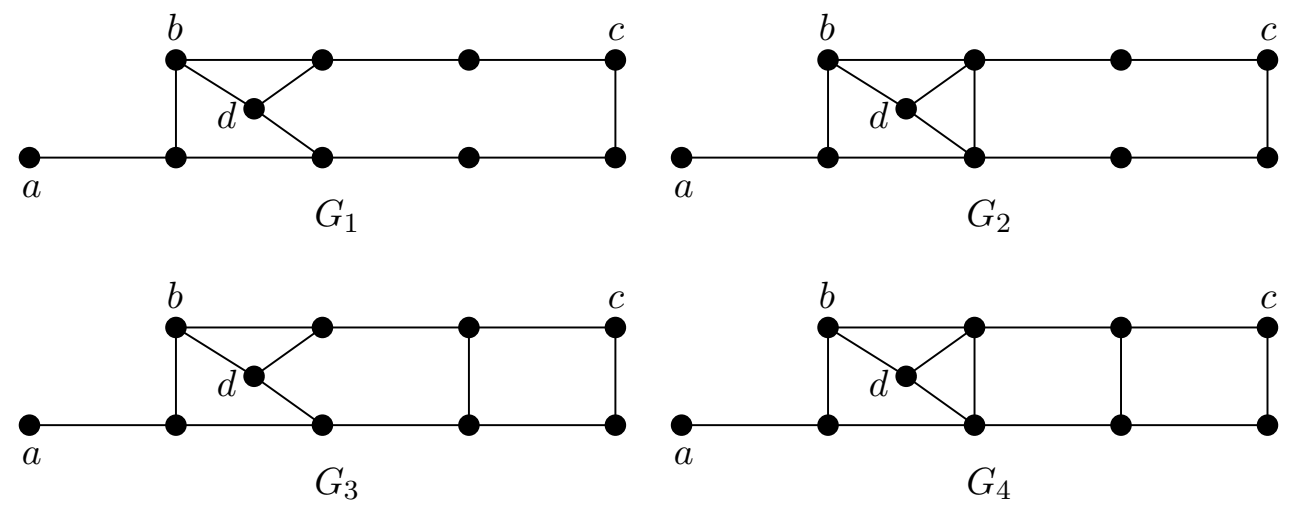

Figura 1: Menores grafos cujo contorno não é geodésico. Em ambos os grafos $G_{i}, C t\left(G_{i}\right)=\{a, b, c\}$ e $I\left[C t\left(G_{i}\right)\right]=V\left(G_{i}\right) \backslash\{d\}$, para $1 \leq i \leq 4$

Observamos que, para todo grafo $G_{i}$ na Figura 1, o vértice $d$ é o único vértice de $G_{i}$ que não pertence a $I\left[C t\left(G_{i}\right)\right]$. Dessa forma, utilizando os Teoremas 1 e 3, obtemos o Corolário 4, que é uma contribuição inicial para o problema proposto em [3], que permanece em aberto, se existe um grafo $G$ tal que $I[I[C t(G)]] \neq V(G)$.

Corolário 4. Se $n \leq 10$, então $I[C t(G)]$ é geodésico.

Palavras-chave: Teoria dos Grafos, Convexidade em Grafos, Análise de Algoritmos

\section{Referências}

[1] D. Artigas, S. Dantas, M.C. Dourado, J.L. Szwarcfiter, and S. Yamaguchi. On the contour of graphs. Discrete Applied Mathematics, 161:1356-1362, 2013.

[2] J. Cáceres, C. Hernando, M. Mora, I. M. Pelayo, M. L. Puertas, and C. Seara. Geodeticity of the contour of chordal graphs. Discrete Applied Mathematics, 156:1132-1142, 2008.

[3] J. Cáceres, A. Márquez, O. R. Oellermann, and M. L. Puertas. Rebuilding convex sets in graphs. Discrete Mathematics, 297:26-37, 2005. 